ments for this disease will quickly ensue from these cutting-edge investigations. The hundreds of thousands of patients with already diagnosed IgA nephropathy and the many more who will be diagnosed with this very common disorder in the future eagerly await these developments.

Address correspondence to: Richard J. Glassock, 8 Bethany, Laguna Niguel, California 92677, USA. Phone: (949) 388-8885; Fax: (949) 388-8882; E-mail: glassock@cox.net.

\footnotetext{
1. Berger, J., and Hinglais, N. 1968. Les depots intercapillaries d'IgA-IgG (Intercapillary deposits of IgA-IgG). J. Urol. Nephrol. (Paris). 74:694-695.

2. D'Amico, G. 1987. The commonest glomerulonephritis in the world: IgA nephropathy. Q. J. Med. 64:709-727.

3. D'Amico, G., et al. 1985. Idiopathic IgA mesangial nephropathy. Clinical and histiological study of 374 patients. Medicine (Baltimore). 64:49-60.

4. Habib, R., Niaudet, P., and Levy, M. 1994. SchonleinHenoch purpura nephritis and IgA nephropathy. In Renal pathology with clinical and functional correlations. C.C. Tisher and B.M. Brenner, editors. J.B. Lippincott \& Co. Philadelphia, Pennsylvania, USA. 472-523.

5. Jozsa, L.G. 2008. Histologic diagnosis of tissue
}

from two nineteenth century Habsburgs. Paleopathol. Newsl. 141:12-18.

6. Conley, M.E., Cooper, M.D., and Michael, A.F. 1980. Selective deposition of immunoglobulin $A_{1}$ in immunoglobulin A nephropathy, anaphylactoid purpura and systemic lupus erythematosus. J. Clin. Invest. 66:1432-1436.

7. Suzuki, H., et al. 2008. IgA1 secreting cell lines from patients with IgA nephropathy produce aberrantly glycosylated IgA1. J. Clin. Invest. 118:629-639.

8. Feehally, J., and Allen, A.C. 1999. Structural features of IgA molecules which contribute to IgA nephropathy. J. Nephrol. 12:59-65.

9. Mestecky, J., et al. 1993. Defective galactosylation and clearance of IgA1 molecules as a possible etiopathogenic factor in IgA nephropathy. Contrib. Nephrol. 104:172-182.

10. Hiki, Y., Iwase, H., and Saitoh, M. 1992. O-linked oligosacchardie (Gal $\beta 1,3$ GalNAc) on IgA hinge region in IgA nephropathy [abstract]. In $\operatorname{IgA}$ nephropathy. The 25th year International Symposium. M.C. Bene, G.C. Faure, and M. Kessler, editors. Karger Medical and Scientific Publishers. Nancy, France/Basel, Switzerland. 222 pp.

11. Hiki, Y., et al. 1995. O-linked oligosaccharide on IgA1 hinge region in IgA nephropathy. Fundamental study for precise structure and possible role. Contrib. Nephrol. 111:73-84.

12. Allen, A.C., Harper, S.J., and Fehally, J. 1995. Galactosylation of $\mathrm{N}$ - and O-linked carbohydrate moieties of IgA1 and IgG in IgA nephropathy. Clin. Exp. Immunol. 100:470-474.
13. Andre, P.M., Le Pogamp, P., and Chevet, D. 1990. Impairment of jacalin binding to serum $\operatorname{IgA}$ in $\operatorname{IgA}$ nephropathy. J. Clin. Lab. Anal. 4:115-119.

14. Moldoveanu, Z., et al. 2007. Patients with IgA nephropathy have increased serum galactose-deficient IgA1 levels. Kidney Int. 71:1148-1154.

15. Novak, J., Julian, B.A., Tomana, M., and Mestecky, J. 2008. IgA glycosylation and IgA immune complexes in the pathogenesis of IgA nephropathy. Semin. Nephrol. 28:78-87.

16. Suzuki, H., et al. 2009. Aberrantly glycosylated IgA1 in IgA nephropathy patients is recognized by IgG antibodies with restricted heterogeneity. J. Clin. Invest. 119:1668-1677.

17. Kokubo, T., et al. 1998. Protective role of IgA1 glycan against IgA1 self-aggregation and adhesion to intracellular matrix proteins. J. Am. Soc. Nephrol. 9:2048-2054.

18. Hebert, L.A., Cosio, F.G., and Birmingham, D.J. 2001. Complement and complement regulatory proteins in renal disease. In Immunologic renal diseases. 2nd edition. E.G. Neilson and W.G. Couser, editors. Lippincott Williams \& Wilkins. Philadelphia, Pennsylvania, USA. 367-394

19. Aucouturier, P., Monteiro, R.C., Noel, L.H., Preud'homme, J.L., and Lesavre, P. 1989. Glomerular and serum immunoglobulin G subclasses in IgA nephropathy. Clin. Immunol. Immunopathol. 51:338-347.

20. Glassock, R.J. Future prospects for IgA nephropathy. 2009. In Advances in IgA nephropathy. K.N. Lai, editor. World Scientific Publishing Co. Singapore. 403-411.

\title{
Integrin-linked kinase: both Jekyll and Hyde in rhabdomyosarcoma
}

\author{
Paul C. McDonald, ${ }^{1}$ Shoukat Dedhar, ${ }^{1,2}$ and Charles Keller ${ }^{3}$
}

\begin{abstract}
1Department of Cancer Genetics, British Columbia Cancer Research Centre, and British Columbia Cancer Agency, Vancouver, British Columbia, Canada. 2Department of Biochemistry and Molecular Biology, University of British Columbia, Vancouver, British Columbia, Canada. ${ }^{3}$ Department of Cellular and Structural Biology and Department of Pediatrics, Greehey Children's Cancer Research Institute, University of Texas Health Science Center, San Antonio, Texas, USA.
\end{abstract}

\begin{abstract}
Although the molecular differences between embryonal rhabdomyosarcoma (ERMS) and alveolar rhabdomyosarcoma (ARMS) have been extensively interrogated, effective therapies tailored to a particular rhabdomyosarcoma subtype have yet to emerge. Patients with ERMS have shown incremental improvement using current multimodal therapy, but survival rates for metastatic ARMS remain poor. In this issue of the JCI, Durbin and colleagues demonstrate that integrin-linked kinase (ILK) acts as a tumor suppressor in ERMS and as a proto-oncogene in ARMS, and that the opposing functions of this enzyme are dependent on the JNK1 signaling pathway (see the related article beginning on page 1558). Their findings suggest that targeting ILK may represent a focused therapeutic strategy for the treatment of ARMS.
\end{abstract}

Conflict of interest: S. Dedhar is a Scientific Consultant for QLT Inc. C. Keller is a cofounder of Numira Biosciences Inc.

Nonstandard abbreviations used: AP-1, activator protein 1; ARMS, alveolar RMS; ERMS, embryonal RMS; FKHR, forkhead homolog in rhabdomyosarcoma; ILK, integrin-linked kinase; $\alpha$-NAC, nascent polypeptideassociated complex and coactivator $\alpha$; PAX3, paired box 3; RMS, rhabdomyosarcoma.

Citation for this article: J. Clin. Invest. 119:1452-1455 (2009). doi:10.1172/JCI39457.
Rhabdomyosarcoma (RMS) is an aggressive muscle cancer and the most common soft-tissue sarcoma of childhood (1). A great paradox lies in the fact that 2 forms of RMS have remarkably different potentials for cure. Wherein embryonal RMS (ERMS) accounts for more than half of RMS cases, the long-term survival for the metastatic form of this disease exceeds $40 \%$ (2). On the other hand, alveolar RMS (ARMS) accounts for one-quarter of RMS cases, but the cure rate for its metastatic form is $20 \%$ or less $(2,3)$. Although the Intergroup Rhabdomyosarcoma Study Group (IRSG) takes histological subtype into account in risk stratification, there is no substantial differentiation between the 2 subtypes with respect to the therapeutic approach, which consists of surgery, chemotherapy, and radiation (4). Despite encouragement that the outcome for ERMS appears to be improving incrementally since the inception of multimodality therapy, the long-term survival for metastatic ARMS has been dismal and largely unchanged for decades $(3,5)$.

\section{Tumor suppressor at times, oncogene at other times}

Integrin-linked kinase (ILK) is well recognized as an oncogenic protein and is 


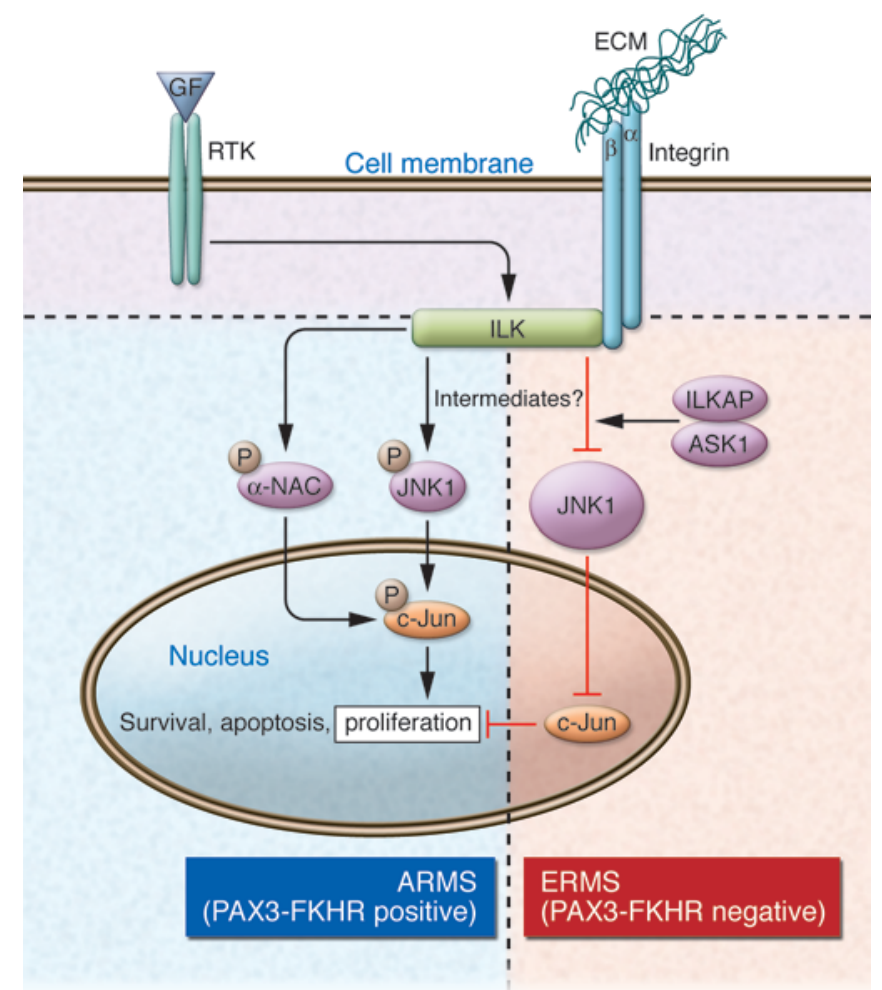

\section{Figure 1}

Differential regulation of JNK by ILK in ARMS versus ERMS. Shown are potential mechanisms of cell growth regulation in ARMS versus ERMS tumor cells related to signaling through ILK, JNK1, and c-Jun. ILK functions are regulated through signals initiated by ECM-integrin interactions or growth factor (GF) stimulation (6). In more clinically favorable cases of ERMS, Durbin et al. (10) demonstrate, in their study in this issue of the $\mathrm{JCl}$, that ILK suppresses phosphorylation of JNK1 and c-Jun, thereby preventing accelerated cell proliferation. While the exact mechanism remains to be shown, the repression of JNK phosphorylation by ILK in this tumor subtype may occur through additional intermediates, including a complex of ILK-associated serine/ threonine phosphatase 2C (ILKAP) with apoptosis signal-regulating kinase 1 (ASK1). In ARMS, JNK1 levels are diminished by the PAX3FKHR oncoprotein, thereby leaving other pathways open to modulating or inducing c-Jun phosphorylation and accelerating cell proliferation. In these tumors, the oncogenic effects of ILK may involve regulation of JNK1 phosphorylation, as suggested by Durbin et al., and/or ILKinduced activation of $\alpha$-NAC. RTK, receptor tyrosine kinase. highly expressed in numerous human cancers, including melanoma, lung, head and neck, pancreas, and prostate cancers $(6,7)$. Importantly, the degree of ILK expression correlates with tumor stage and grade in many of these malignancies, and strong ILK expression is often a poor prognostic indicator $(8,9)$. Previous work has defined ILK as a key regulator of cellular events critical to cancer progression, including proliferation, survival, adhesion, migration, invasion, and angiogenesis $(6,7)$. The inhibition of ILK expression and/or activity using genetic and pharmacologic strategies has established the requirement of ILK for tumor growth. The necessity of ILK for cancer progression, particularly in carcinomas, has centered on the capacity of ILK to phosphorylate and regulate downstream signaling targets of the PI3K pathway, notably Akt and glycogen synthase kinase-3 $\beta(6,7)$. ILK functionality is dependent on cellular context, however, and ILK is reported to suppress growth in certain circumstances (6).

In this issue of the JCI, Durbin et al. (10) present one set of fundamental biological differences between ERMS and ARMS: the levels of ILK, JNK1, and the phosphorylated JNK1 protein. The authors used a series of model systems, including cultured human cells, murine xenografts, and primary human tumors, to demonstrate that ILK functions as a tumor suppressor in ERMS, whereas it acts as an oncogene in ARMS. In contrast to many cancer models, Akt or MAPK signaling remained unaffected by inhibition of ILK, and the opposing functions of ILK in these tumors were attributed to the presence of a noncanonical target of ILK, JNK1 (Figure 1). This work is intriguing because it attempts to provide a mechanistic basis for the oncogenic versus tumorsuppressive functions attributed to ILK. In their report, Durbin et al. focus on JNK1 as a mediator of proliferation (10) - yet much of the literature highlights the role of JNK as a proapoptotic factor that stabilizes p53 or promotes apoptosis by c-Jun-mediated transcription of proapoptotic genes (11). What biology underlies the difference in JNK activity in these 2 subtypes of RMS? An intriguing possibility may lie in the ratio between ILK and JNK, wherein low JNK levels result in c-Jun-mediated survival and proliferation, but high and sustained JNK (and c-Jun) levels lead to apoptosis.

\section{Genetics could explain how tumors tip the balance}

Durbin et al. showed that for ERMS, ILK expression resulted in inhibition of JNK phosphorylation, possibly through one or more intermediate effectors (10). siRNAmediated depletion of ILK reversed the suppression of JNK phosphorylation and led to phosphorylation of c-Jun. Phospho-c-Jun, as a presumed component of a heterodimeric activator protein 1 (AP-1) complex, regulates cell proliferation. Consistent with the role of ILK as a tumor suppressor in ERMS, the authors report that ILK expression in primary tumor samples from patients with invasive and metastatic ERMS in unfavorable anatomical locations (i.e., stage III or IV at diagnosis) was significantly reduced compared with less invasive or less metastatic ERMS in more favorable anatomical locations (i.e., stage I or II at diagnosis). ILK expression was also positively associated with survival for ERMS patients at all disease stages at diagnosis. Durbin et al. point out that chromosomal locus $11 \mathrm{p} 15$ is a frequent site of loss of imprinting/loss of heterozygosity in ERMS, and this region also contains the ILK gene (10). In ERMS, p53 loss of function is also not uncommon - indeed, familial $p 53$ haploinsufficiency, or Li-Fraumeni syndrome, was a description of familial ERMS (12). Therefore, could loss of the ILK locus for high-stage ERMS lead to increased levels of phospho-JNK, and could concurrent p53 
loss result in phospho-JNK-mediated c-Jun phosphorylation and proliferation, instead of phospho-JNK/p53-mediated apoptosis? This speculative scenario is one possible way to integrate the present findings into the genetics defined to date for ERMS.

In contrast to their results for ERMS, Durbin et al. show in mouse xenografts that ILK functioned as an oncogene in ARMS and that ILK inhibition slowed tumor growth (10). Previous work has shown that $55 \%$ or more of ARMS patients harbor a translocation-mediated chimeric oncogene resulting from the fusion of paired box 3 $(P A X 3)$ to forkhead homolog in rhabdomyosarcoma (FKHR, also known as FOXO1A; ref. 13). Introduction of the PAX3-FKHR chimeric transcription factor into ERMS cells resulted in decreased JNK1B1 transcript levels. In these PAX3-FKHR-expressing ERMS cells, siRNA-mediated suppression of ILK expression resulted in decreased tumor cell growth in vitro, similar to the behavior of ARMS cells when ILK was experimentally depleted (10). The authors further showed that restoration of JNK1 protein in ARMS led to a situation more like ERMS, in which decreased ILK expression led to increased tumor cell growth. It may be that ARMS cells escape phospho-JNK/p53-mediated apoptosis in this situation because p53 loss of function is also not uncommon in ARMS (14). Thus, JNK1 protein levels appear to be at the crux of whether ILK acts as a tumor suppressor in ERMS or as an oncogene in ARMS (Figure 1).

There is perhaps a common biological thread connecting human ERMS and ARMS tumors. In the studies by Durbin and colleagues (10), both ERMS cells with experimentally decreased levels of ILK (i.e., diminished tumor suppression) and ARMS cells with native levels of oncogenic ILK exhibited elevated levels of phosphoc-Jun. In their work, a dominant-negative c-Jun mutant suppressed tumor cell growth in the former instance (10), and might be assumed to do the same in the latter, which suggests that phospho-c-Jun is helping to drive tumor growth in ARMS and clinical stage III/IV ERMS. Paradoxically, sustained and very elevated levels of c-Jun, as seen in response to the immunosuppressant/ mammalian target of rapamycin inhibitor rapamycin, leads to c-Jun-mediated apoptosis when p53 is absent (15). Therefore, phospho-c-Jun, as a component of an AP-1 complex, may act as a proliferative factor when present at levels below a particular threshold; however, high and sustained levels of phospho-c-Jun, as found during periods of cellular stress, can lead to apoptosis of cells. While it is beyond the scope of this commentary to address AP-1 signaling (reviewed in ref. 16), a closer examination of c-Jun heterodimerization partners (e.g., c-Fos and JunB) under conditions of stress in ERMS and ARMS may be warranted.

\section{Unanswered questions}

The study by Durbin et al. (10) raises a number of important questions that remain to be resolved. The authors provide compelling evidence of the Jekyll and Hyde nature of ILK: its tumor-suppressive function in ERMS and its oncogenic role in ARMS. A key issue, however, is the mechanism by which ILK targets JNK activity. How does ILK function to suppress JNK activation in ERMS, yet activate JNK in ARMS? Initial investigations did not demonstrate the presence of a direct interaction between ILK and JNK (10), leaving open the possibility that additional, as-yet-unidentified proteins are required for effective signaling between ILK and JNK. One potential mediator is ILK-associated serine/threonine phosphatase $2 \mathrm{C}$, which is known to associate not only with ILK, but also with an upstream activator of JNK called apoptosis signal-regulating kinase 1 (Figure 1 and ref. 17). It is also possible that part of the effect of ILK on JNK and c-Jun is mediated by ILK-induced activation of the c-Jun transcriptional coactivator nascent polypeptide-associated complex and coactivator $\alpha$ $(\alpha$-NAC; Figure 1$)$. It has previously been shown that ILK phosphorylates $\alpha$-NAC at serine 43 , resulting in its nuclear accumulation and potentiation of c-Jun-mediated transcriptional regulation (18).

Durbin et al. used siRNA to transiently suppress ILK expression in the ERMS and ARMS cell lines prior to the inoculation of these cells into a xenograft model (10). Importantly, the cell lines were implanted 3 days after introduction of the siRNA into the cells, a time point at which ILK expression was effectively inhibited (10). However, the short-term depletion of target gene expression afforded by the use of siRNA, in contrast to the stable knockdown that can be achieved using shRNA technologies, leaves open to debate the precise role of ILK in tumor growth in this model. Certainly, depletion of ILK expression prior to introduction of the ERMS and ARMS cells into the mouse xenograft model resulted in striking and sustained effects on tumor growth, as well as on apoptosis and angiogenesis. However, ILK expression ultimately returned in both tumor groups, which suggests that ILK depletion may be critical as much for early tumor establishment as for signaling to the JNK pathway during growth. The use of shRNA to stably depress ILK levels and/or the use of inducible systems to provide native ILK expression during tumor establishment may help to further address this issue.

\section{Returning to the bedside}

The data presented by Durbin et al. (10) suggest that prospective evaluation of the ILK/JNK1/c-Jun signaling axis may be worthwhile in the pursuit of molecular diagnostics for risk stratification of RMS patients at diagnosis. More importantly, these studies allude to what we believe to be a novel therapeutic strategy for the treatment of ARMS - the inhibition of the activity or expression of ILK. This can be accomplished with small-molecule ILK inhibitors that have been extensively characterized in preclinical models of several types of cancers (reviewed in refs. 6, 7). In addition, genetic strategies involving silencing ILK expression can also be considered, given the advances in shRNA and siRNA delivery strategies. However, the effects of ILK inhibitors would be untoward in ERMS. Given the high rate of misdiagnosis of ARMS versus ERMS (as high as 37\%; refs. $4,19)$ and the possibility that ILK signaling may be different under nascent versus stressed conditions such as chemotherapy, it may be well worth additional time in the laboratory to understand the basis of this intriguing difference in ILK behavior in RMS subtypes.

Address correspondence to: Charles Keller, University of Texas Health Science Center at San Antonio, 8403 Floyd Curl Drive, MC-7784, San Antonio, Texas 78229-3900, USA. Phone: (210) 562-9062; Fax: (210) 562-9014; E-mail: kellerc2@uthscsa.edu.

1. Arndt, C.A., and Crist, W.M. 1999. Common musculoskeletal tumors of childhood and adolescence. N. Engl. J. Med. 341:342-352.

2. Breneman, J.C., et al. 2003. Prognostic factors and clinical outcomes in children and adolescents with metastatic rhabdomyosarcoma - a report from the Intergroup Rhabdomyosarcoma Study IV. J. Clin. Oncol. 21:78-84.

3. Williams, B.A., et al. 2004. Metastatic rhabdomyosarcoma: a retrospective review of patients treated at the hospital for sick children between 1989 and 1999. J. Pediatr. Hematol. Oncol. 26:243-247.

4. Raney, R.B., et al. 2001. The Intergroup Rhabdomyosarcoma Study Group (IRSG): Major lessons from the IRS-I through IRS-IV studies as background for the current IRS-V treatment protocols. 
Sarcoma. 5:9-15.

5. Maurer, H.M., et al. 1988. The Intergroup Rhabdomyosarcoma Study - I. A final report. Cancer. 61:209-220.

6. McDonald, P.C., Fielding, A.B., and Dedhar, S. 2008 Essential roles of integrin-linked kinase in physiology and cancer biology. J. Cell. Sci. 121:3121-3132.

7. Hannigan, G., Troussard, A.A., and Dedhar, S. 2005 Integrin-linked kinase: a cancer therapeutic target unique among its ILK. Nat. Rev. Cancer. 5:51-63.

8. Yau, C.Y., Wheeler, J.J., Sutton, K.L., and Hedley, D.W. 2005. Inhibition of integrin-linked kinase by a selective small molecule inhibitor, QLT0254, inhibits the PI3K/PKB/mTOR, Stat3, and FKHR pathways and tumor growth, and enhances gemcitabine-induced apoptosis in human orthotopic primary pancreatic cancer xenografts. Cancer Res. 65:1497-1504.

9. Takanami, I. 2005. Increased expression of integ- rin-linked kinase is associated with shorter survival in non-small cell lung cancer. BMC Cancer. 5:1.

10. Durbin, A.D., et al. 2009. JNK1 determines the oncogenic or tumor-suppressive activity of the integrin-linked kinase in human rhabdomyosarcoma. J. Clin. Invest. 119:1558-1570.

11. Dhanasekaran, D.N., and Reddy, E.P. 2008. JNK signaling in apoptosis. Oncogene. 27:6245-6251.

12. Li, F.P., and Fraumeni, J.F., Jr. 1969. Rhabdomyosarcoma in children: epidemiologic study and identification of a familial cancer syndrome. J. Natl. Cancer Inst. 43:1365-1373.

13. Sorensen, P.H., et al. 2002. PAX3-FKHR and PAX7FKHR gene fusions are prognostic indicators in alveolar rhabdomyosarcoma: a report from the children's oncology group. J. Clin. Oncol. 20:2672-2679.

14. Keller, C., et al. 2004. Alveolar rhabdomyosarcomas in conditional Pax3:Fkhr mice: cooperativity of Ink4a/ARF and Trp53 loss of function. Genes Dev.
18:2614-2626.

15. Huang, S., et al. 2003. Sustained activation of the JNK cascade and rapamycin-induced apoptosis are suppressed by $\mathrm{p} 53 / \mathrm{p} 21$ (Cip1). Mol. Cell. 11:1491-1501

16. Shaulian, E., and Karin, M. 2002. AP-1 as a regulator of cell life and death. Nat. Cell. Biol. 4:E131-E136.

17. Tamura, S., et al. 2006. PP2C family members play key roles in regulation of cell survival and apoptosis. Cancer Sci. 97:563-567.

18. Quelo, I., Gauthier, C., Hannigan, G.E., Dedhar, S., and St-Arnaud, R. 2004. Integrin-linked Kinase Regulates the Nuclear Entry of the c-Jun Coactivator \{alpha\}-NAC and Its Coactivation Potency. J. Biol. Chem. 279:43893-43899.

19. Carli, M., et al. 2004. European intergroup studies (MMT4-89 and MMT4-91) on childhood metastatic rhabdomyosarcoma: final results and analysis of prognostic factors. J. Clin. Oncol. 22:4787-4794.

\title{
Infantile parkinsonism-dystonia: a dopamine "transportopathy"
}

\author{
Craig Blackstone
}

Cellular Neurology Unit, Neurogenetics Branch, National Institute of Neurological Disorders and Stroke, NIH, Bethesda, Maryland, USA.

The dopamine transporter (DAT) retrieves the neurotransmitter dopamine from the synaptic cleft at dopaminergic synapses. Variations in solute carrier family 6A, member 3 (SLC6A3/DAT1), the human gene encoding DAT, have been implicated in attention deficit hyperactivity and bipolar disorders, and DAT is a prominent site of action for drugs such as amphetamines and cocaine. In this issue of the JCI, Kurian et al. report that an autosomal recessive infantile parkinsonism-dystonia is caused by loss-of-function mutations in DAT that impair dopamine reuptake (see the related article beginning on page 1595). Though this might be predicted to result in dopamine excess in the synaptic cleft, it likely also causes depletion of presynaptic dopamine stores and possibly downregulation of postsynaptic dopamine receptor function, resulting in impairments in dopaminergic neurotransmission consistent with the clinical presentation. This is the first report of a genetic alteration in DAT function underlying a parkinsonian disorder.

The complex interconnections in the nervous system depend on synapses, specialized junctions at which a neuron contacts a target cell, most frequently another neuron. Synaptic transmission is mediated by chemical neurotransmitters in the synaptic cleft ( 20-25 $\mathrm{nm}$ wide) and is regulated dynamically by presynaptic neurotransmitter release and subsequent reuptake as well as by other pre- and postsynaptic signaling mechanisms. Many neurotransmit-

Conflict of interest: The author has declared that no conflict of interest exists.

Nonstandard abbreviations used: DAT, dopamine transporter; SLC6, solute carrier family 6; SLC6A3, SLC6A, member 3; TM, transmembrane domain.

Citation for this article: J. Clin. Invest. 119:1455-1458 (2009). doi:10.1172/JCI39632. ter receptors are ligand-gated ion channels, including the predominant postsynaptic excitatory and inhibitory receptors in the central nervous system, which are gated by glutamate and GABA, respectively. Other neurotransmitter receptors are coupled through $G$ proteins to intracellular second messenger systems, including receptors for the biogenic amine dopamine.

Dopaminergic neurons are found in relatively restricted areas in the brain, but prominently within the substantia nigra pars compacta, with projections to striatum, and in the ventral tegmental area of the midbrain, with projections to the cerebral cortex as well as to limbic and subcortical nuclei. Consistent with this distribution, dopamine exerts modula- tory effects on human motor control, affect, behavior, and cognition $(1,2)$. Alterations in dopaminergic transmission have been implicated in a host of neuropsychiatric and movement disorders (3). These comprise both dopamine deficiency states such as Parkinson disease as well as states of dopamine excess, as proposed for Tourette syndrome and schizophrenia. Furthermore, a large number of commonly prescribed neuropsychiatric medications as well as drugs of abuse have major effects at these synapses. For instance, L-dopa used to treat Parkinson disease, methylphenidate and amphetamine used for attention deficit hyperactivity disorder, and cocaine all promote dopaminergic transmission, while neuroleptic medications commonly prescribed for schizophrenia and Tourette syndrome inhibit dopamine transmission.

The dopaminergic synapse comprises a number of signature elements, situated both pre- and postsynaptically (Figure 1A). Postsynaptic dopamine receptors can be divided into two major subtypes that are positively (D1 class) or negatively (D2 class) coupled through $\mathrm{G}$ proteins to regulate production of cAMP by adenylate cyclase. D1-class receptors are also coupled to phosphoinositide metabolism, and D2class receptors are coupled to $\mathrm{Ca}^{2+}$-dependent intracellular signaling cascades (4). 